\title{
An accurate numerical method for solving the general- ized time-fractional diffusion equation
}

\author{
Muhammed Syam ${ }^{a, *}$, Ibrahim Al-Subaihib \\ ${ }^{a}$ Department of Mathematical Sciences, United Arab Emirates University, Al Ain, UAE. \\ ${ }^{b}$ Department of Mathematics, Taibah University, Al Madinah, Saudi Arabia.
}

Communicated by R. Saadati

\begin{abstract}
In this paper, a formulation for the fractional Legendre functions is constructed to solve a class of time-fractional diffusion equation. The fractional derivative is described in the Caputo sense. The method is based on the collection Legendre. Analysis for the presented method is given and numerical results are presented.
\end{abstract}

Keywords: Fractional-order Legendre function, collocation method, generalized time-fractional diffusion equation.

2010 MSC: 34A08, 34Bxx, 35J40, 47H10, 65L05.

(C)2018 All rights reserved.

\section{Introduction}

Fractional diffusion-wave equation has been used widely in many branches of science and engineering. These equations represent propagation of mechanical waves in visco-elastic media $[7,9,10]$, a nonMarkovian diffusion process with memory [11], charge transport in amorphous semiconductors [12] and many more. In $[7,9,10]$, they studied the fractional wave equation governing the propagation of mechanical diffusive waves in viscoelastic media which exhibit a power-law creep. Gejji and Bhalekar [4] used fractional diffusion-wave equation to model electromagnetic acoustic, and mechanical responses. Luchko [12] investigated a continuous time random walks on fractals. Mainardi [13] studied the relaxation phenomena in complex viscoelastic material using fractional diffusion equations.

Various methods such as Green's function method [4], finite sine trans- form method [15], method of images and Fourier transform [11], separation of variables method [17], finite difference method [18], Adomian decomposition method (ADM) $[19,20]$ have been used to solve these equations. Recently, Al-Mdallal and Syam [2] have devised a New Iterative Method (NIM) to solve non-linear functional equations. This method is free from rounding of errors since it does not involve discretization and has

\footnotetext{
${ }^{*}$ Corresponding author

Email address: m.syam@uaeu.ac . ae (Muhammed Syam)

doi: 10.22436/jnsa.011.11.08
} 
fairly simple algorithm. Also it does not require prior knowledge of the concepts such as Lagrange multipliers or homotopy. More methods can be found in [1, 3, 5, 6, 8, 14, 23-25].

We consider a class of the time-fractional diffusion equation of the form

$$
D_{t}^{\alpha} u(x, t)=a(x, t) D_{x}^{\beta} u(x, t)+f(x, t), \quad x \in(0,1), \quad t \in(0, T),
$$

with initial and boundary conditions

$$
u(0, t)=h_{1}(t), u(1, t)=h_{2}(t) \text { and } u(x, 0)=g(x),
$$

where $a, f \in C^{1}([0,1] \times[0, T]), g \in C[0,1], h_{1}, h_{2} \in C[0, T], T>0,1<\beta \leqslant 2$, and $0<\alpha \leqslant 1$. For $\alpha=1$ and $\beta=2$, the fractional diffusion equation is reduced to a conventional diffusion-reaction equation which is well studied, so we face on $0<\alpha<1$.

\section{Preliminaries}

In this section, we present the definition and some preliminary results of the Caputo fractional derivatives, as well as, the definition of the fractional-order Legendre functions and their properties.

Definition 2.1. A real function $f(t), t>0$, is said to be in the space $C_{\mu}, \mu \in \mathbb{R}$ if there exists a real number $p>\mu$, such that $f(t)=t^{p} f_{1}(t)$, where $f_{1}(t) \in C[0, \infty)$, and it is said to be in the space $C_{\mu}^{m}$ if $f^{(m)} \in C_{\mu}, m \in \mathbb{N}$.

Definition 2.2. The left Riemann-Liouville fractional integral of order $\delta \geqslant 0$, of a function $f \in C_{\mu}, \mu \geqslant-1$, is defined by

$$
I^{\delta} f(t)= \begin{cases}\frac{1}{\Gamma(\delta)} \int_{0}^{t}(t-s)^{\delta-1} f(s) d s, & \delta>0 \\ f(t), & \delta=0 .\end{cases}
$$

Definition 2.3. For $\delta>0, m-1<\delta<m, m \in \mathbb{N}, t>0$, and $f \in C_{-1}^{m}$, the left Caputo fractional derivative is defined by

$$
D^{\delta} f(t)= \begin{cases}\frac{1}{\Gamma(m-\delta)} \int_{0}^{t}(t-s)^{m-1-\delta} f^{(m)}(s) d s, & \delta>0, \\ f^{\prime}(t), & \delta=0,\end{cases}
$$

where $\Gamma$ is the well-known Gamma function.

The Caputo derivative defined in (2.1) is related to the Riemann-Liouville fractional integral, $\mathrm{I}^{\delta}$, of order $\delta \in \mathbb{R}^{+}$, by $D^{\delta} f(t)=I^{m-\delta} f^{(m)}(t)$. The Caputo fractional derivative satisfy the following properties for $f \in C_{\mu}, \mu \geqslant-1$ and $\gamma \geqslant 0$, see [16].

1. $D^{\gamma} I^{\gamma} f(t)=f(t)$

2. $I^{\gamma} D^{\gamma} f(t)=f(t)-\sum_{k=0}^{n-1} f^{(k)}(0) \frac{t^{k}}{k !} ;$

3. $\mathrm{D}^{\gamma} \mathrm{c}=0$, where $\mathrm{c}$ is constant;

4. $\mathrm{D}^{\gamma} \mathrm{t}^{\mu}=\left\{\begin{array}{ll}0, & \mu<\gamma, \mu \in\{0,1,2, \ldots\}, \\ \frac{\Gamma(\mu+1)}{\Gamma(\mu-\gamma+1)} \mathrm{t}^{\mu-\gamma}, & \text { otherwise, }\end{array}\right\}$;

5. $D^{\gamma}\left(\sum_{k=0}^{m} c_{i} f_{i}(t)\right)=\sum_{k=0}^{m} c_{i} D^{\gamma} f_{i}(t)$, where $c_{1}, c_{2}, \ldots, c_{m}$ are constants.

The basic concept of this paper is the Legendre polynomials. For this reason, we study some of their properties.

Definition 2.4. The Legendre polynomials $\left\{L_{k}(x): k=0,1,2, \ldots\right\}$ are the eigenfunctions of the SturmLiouville problem

$$
\left(\left(1-x^{2}\right) \mathrm{L}_{\mathrm{k}}^{\prime}(x)\right)^{\prime}+\mathrm{k}(\mathrm{k}+1) \mathrm{L}_{\mathrm{k}}(x)=0, \quad x \in[-1,1] .
$$


Among the properties of the Legendre polynomials we list the following [21]:

1. $\int_{-1}^{1} L_{i}(x) L_{j}(x) d x=\frac{2}{2 i+1} \delta_{i j}$, where $\delta_{i j}=\left\{\begin{array}{ll}0, & i \neq j, \\ 1, & i=j,\end{array}\right\}$;

2. $L_{i+1}(x)=\frac{2 i+1}{i+1} x L_{i}(x)-\frac{i}{i+1} L_{i-1}(x)$ for $i \geqslant 1$;

3. $\mathrm{L}_{\mathfrak{i}}( \pm 1)=( \pm 1)^{i}$.

One of the common and efficient methods for solving fractional differential equations of order $\gamma>0$ is using series expansion of the form $\sum_{k=0}^{i} c_{k} t^{\gamma k}$. For this reason, we define the fractional-order Legendre function by $\mathrm{F}_{i}^{\gamma}(\mathrm{t})=\mathrm{L}_{i}\left(2 \mathrm{t}^{\gamma}-1\right)$. Using the properties of the Legendre polynomials, it is easy to verify that ([22])

1. $\left(\left(t-t^{1+\gamma}\right) F_{i}^{\gamma^{\prime}}(t)\right)^{\prime}+i(i+1) \gamma^{2} t^{\gamma-1} F_{i}^{\gamma}(t)=0, t \in(0,1) ;$

2. $F_{i}^{\gamma}(0)=(-1)^{i}$ and $F_{i}^{\gamma}(1)=1$.

In addition, $\left\{\mathrm{F}_{i}^{\gamma}(\mathrm{t}): i=0,1,2, \ldots\right\}$ are orthogonal functions with respect to the weight function $w(t)=$ $t^{\gamma-1}$ on $(0,1)$ with

$$
\int_{0}^{1} F_{i}^{\gamma}(t) F_{j}^{\gamma}(t) w(t) d t=\frac{1}{(2 i+1) \gamma} \delta_{i j} .
$$

The closed form of $F_{i}^{\gamma}(t)$ is given by

$$
F_{i}^{\gamma}(t)=\sum_{k=0}^{i}(-1)^{i+k} \frac{(i+k) !}{(i-k) !} \frac{t^{\gamma k}}{(k !)^{2}} .
$$

Using properties (4) and (5) of the Caputo fractional derivative, one can see that

$$
D^{\gamma} F_{i}^{\gamma}(t)=\sum_{k=1}^{i}(-1)^{i+k} \frac{(i+k) !}{(i-k) !(k !)^{2}} \frac{\Gamma(k \gamma+1)}{\Gamma((k-1) \gamma+1)} t^{(k-1) \gamma} .
$$

The following result is important, sine it facilitates applying the collection method.

Theorem 2.5. Let $u \in C^{n}[0,1]$ and $u^{(n+1)}(t)$ be a piecewise continuous function on $[0,1]$ where $n$ is a positive integer greater than or equal 1 . Then,

(i) $\mathrm{u}(\mathrm{t})$ can be written in the infinite expansion as $\mathrm{u}(\mathrm{t})=\sum_{\mathrm{k}=0}^{\infty} \mathrm{u}_{\mathrm{k}} \mathrm{F}_{\mathrm{k}}^{\gamma}(\mathrm{t})$, where

$$
u_{k}=(2 i+1) F_{k}^{\gamma}(t) \int_{0}^{1} u(t) F_{k}^{\gamma}(t) w(t) d t
$$

(ii) $\sum_{k=0}^{\infty} u_{k}^{(\gamma)} F_{k}^{\alpha}(t)$ converges uniformly on $[0,1]$ to $D^{\gamma} u(t)$, where $n-1<\gamma \leqslant n, u_{k}^{(\gamma)}=\sum_{j=k+1}^{\infty} a_{j k} u_{j}$, and $\mathrm{a}_{\mathrm{jk}}=(2 \mathrm{k}+1) \gamma \int_{0}^{1} \mathrm{D}^{\gamma} \mathrm{F}_{j}^{\gamma}(\mathrm{t}) \mathrm{F}_{\mathrm{k}}^{\gamma}(\mathrm{t}) w(\mathrm{t}) \mathrm{dt}$, for $\mathrm{k}=0,1,2, \ldots$ and $\mathrm{j}=\mathrm{k}+1, \mathrm{k}+2, \ldots$

Proof.

(i) Since $u \in C^{1}[-1,1]$ and $u^{\prime \prime}(x)$ is a piecewise continuous function on $[-1,1], \sum_{k=0}^{\infty} v_{k} \mathcal{L}_{k}(x)$ converges uniformly to $u(x)$ on $[-1,1]$, where $\left\{v_{k}\right\}$ can be computed by the orthogonality relation of the Legendre polynomials, see [20]. Since $r:[0,1] \rightarrow[-1,1]$ defined by $r(t)=2 t^{\gamma}-1$, is a bijection continuous function, $\sum_{k=0}^{\infty} u_{k} F_{k}^{\gamma}(t)$ converges uniformly to $u(t)$ on $[0,1]$, where the value of $u_{k}$ follows from the orthogonality relation of $\left\{F_{i}^{\gamma}(t): i=0,1,2, \ldots\right\}$ with respect to the weight function $w(t)=t^{\gamma-1}$ on $[0,1]$, which completes the proof. 
(ii) Let $P_{m}(t)=\sum_{k=0}^{m} u_{k} F_{k}^{\gamma}(t)$ for $n=0,1,2, \ldots$. From Part (i), $P_{n}(t)$ converges uniformly to $u(t)$ on $[0,1]$. Since $u \in C^{n}[0,1]$ and $u^{(n+1) \prime}(t)$ is a piecewise continuous function on $[0,1]$,

$$
\frac{d}{d t}\left(\operatorname{Lim}_{m \rightarrow \infty} P_{m}(t)\right)=\operatorname{Lim}_{m \rightarrow \infty}\left(\frac{d}{d t} P_{m}(t)\right)
$$

and $\frac{d}{d t} P_{m}(t)$ converges uniformly to $\frac{d}{d t} u(t)$ on $[0,1]$. Thus, $\int_{0}^{x} \frac{P_{m}^{(n)}(t)}{(x-t)^{\gamma}} d t$ converges uniformly to $\int_{0}^{x} \frac{u^{(n)}(t)}{(x-t)^{\gamma}} d t$ on $[0,1]$ which gives the result of the second part. The value of $a_{j k}$ follows from the orthogonality relation of $\left\{F_{i}^{\gamma}(t): i=0,1,2, \ldots\right\}$ with respect to the weight function $w(t)=t^{\gamma-1}$ on $[0,1]$.

\section{Collocation method}

In this section, we use the fractional-order Legendre collocation method to discretize Problem (1.1)(1.2). For simplicity, we assume that $T=1$. If $T \neq 1$, we use the change of variable $\tau=\frac{t}{T}$ to make the $\mathrm{t}$-domain $(0,1)$. Expand the solution $u(x, t)$ in terms of the fractional-order Legendre function as follows:

$$
\mathrm{u}_{\mathrm{N}}(x, \mathrm{t})=\sum_{\mathrm{k}=0}^{\mathrm{N}+2} \mathrm{u}_{\mathrm{k}}(\mathrm{t}) \mathrm{F}_{\mathrm{k}}^{\beta}(\mathrm{x})
$$

Thus,

$$
D_{x}^{\beta} u_{N}(x, t)=\sum_{k=0}^{N} u_{k}^{(\beta)}(t) F_{k}^{\beta}(x),
$$

where $u_{k}^{(\beta)}=\sum_{j=k+1}^{\infty} b_{j k} u_{j}$, and $b_{j k}=(2 k+1) \beta \int_{0}^{1} D^{\beta} F_{j}^{\beta}(x) F_{k}^{\beta}(x) w(t) d t$ for $k=0,1,2, \ldots$ and $j=$ $k+1, k+2, \ldots$ for $k=0,1, \ldots, N$. Therefore, for $U_{N}(x, t)$, the residual is given by

$$
R\left(U_{N}(x, t)\right) \approx D_{t}^{\alpha} U_{N}(x, t)-a(x, t) D_{x}^{\beta} U_{N}(x, t)-f(x, t) .
$$

Orthogonalized the residual with respect to the Dirac delta function as follows:

$$
<R\left(U_{N}(x, t)\right), \delta\left(x-x_{j}\right)>=\int_{0}^{1} R\left(U_{N}(x, t)\right) \delta\left(x-x_{j}\right) d x=0, \text { for } j=0: N,
$$

where $x_{j}$ are the collocation points. We choose the collocation points to be the roots of $\mathcal{L}_{N+2}^{\prime}$. Therefore, equation (3.2) leads to the elementwise equation

$$
D_{t}^{\alpha} U_{N}\left(x_{j}, t\right)-a\left(x_{j}, t\right) D_{x}^{\beta} U_{N}\left(x_{j}, t\right)+f\left(x_{j}, t\right)=0, \quad \text { for } j=0: N,
$$

or

$$
\sum_{k=0}^{N+2}\left[D_{t}^{\alpha} u_{k}(t)\right] F_{k}^{\beta}\left(x_{j}\right)-a\left(x_{j}, t\right) \sum_{k=0}^{N} u_{k}^{(2)}(t) F_{k}^{\beta}\left(x_{j}\right)-f\left(x_{j}, t\right)=0, \quad \text { for } j=0: N
$$

Let

$$
u(t)=\left[\begin{array}{c}
u_{0}(t) \\
u_{1}(t) \\
\vdots \\
u_{N+2}(t)
\end{array}\right], U^{(\beta)}(t)=\left[\begin{array}{c}
u_{0}^{(\beta)}(t) \\
u_{1}^{(\beta)}(t) \\
\vdots \\
u_{N}^{(\beta)}(t)
\end{array}\right], F(t)=\left[\begin{array}{c}
f\left(x_{0}, t\right) \\
f\left(x_{1}, t\right) \\
\vdots \\
f\left(x_{N}, t\right)
\end{array}\right]
$$


Thus, we can rewrite equation (3.3) in the matrix form as

$$
A_{1} D_{t}^{\alpha} U(t)-A(t) A_{2} U^{(2)}(t)-F(t)=0,
$$

where

$$
\begin{aligned}
A_{1} & =\left[\begin{array}{cccc}
F_{0}^{\beta}\left(x_{0}\right) & F_{1}^{\beta}\left(x_{0}\right) & \ldots & F_{N+2}^{\beta}\left(x_{0}\right) \\
F_{0}^{\beta}\left(x_{1}\right) & F_{1}^{\beta}\left(x_{1}\right) & \ldots & F_{N+2}^{\beta}\left(x_{1}\right) \\
\vdots & \vdots & \ddots & \vdots \\
F_{0}^{\beta}\left(x_{N}\right) & F_{1}^{\beta}\left(x_{N}\right) & \ldots & F_{N+2}^{\beta}\left(x_{N}\right)
\end{array}\right], \\
A & =\left[\begin{array}{cccc}
a\left(t, x_{0}\right) & 0 & \ldots & 0 \\
0 & a\left(t, x_{1}\right) & \ldots & 0 \\
\vdots & \vdots & \ddots & \vdots \\
0 & 0 & \ldots & a\left(t, x_{N}\right)
\end{array}\right], \quad A_{2}=\left[\begin{array}{cccc}
F_{0}^{\beta}\left(x_{0}\right) & F_{1}^{\beta}\left(x_{0}\right) & \ldots & F_{N}^{\beta}\left(x_{0}\right) \\
F_{0}^{\beta}\left(x_{1}\right) & F_{1}^{\beta}\left(x_{1}\right) & \ldots & F_{N}^{\beta}\left(x_{1}\right) \\
\vdots & \vdots & \ddots & \vdots \\
F_{0}^{\beta}\left(x_{N}\right) & F_{1}^{\beta}\left(x_{N}\right) & \ldots & F_{N}^{\beta}\left(x_{N}\right)
\end{array}\right] .
\end{aligned}
$$

Since $u_{k}^{(\beta)}=u_{k}^{(\beta)}=\sum_{j=k+1}^{\infty} b_{j k} u_{j}$, and $b_{j k}=(2 k+1) \beta \int_{0}^{1} D^{\beta} F_{j}^{\beta}(x) F_{k}^{\beta}(x) w(t) d t$, for $k=0,1,2, \ldots$ and $j=k+1, k+2, \ldots$ for $k=0,1, \ldots, N$, it is easy to see that $U^{(\beta)}(t)=A_{3} U(t)$, where $A_{3}$ is $(N+1) \times(N+3)$ matrix. Therefore, system (3.4) becomes

$$
A_{1} D_{t}^{\alpha} U(t)-A(t) A_{2} A_{3} U(t)-F(t)=0 .
$$

Now, we study the boundary conditions on the variable $x$. From equation (3.1), one can see that

$$
\left.\left.\mathrm{u}_{\mathrm{N}}(0, \mathrm{t})=\sum_{k=0}^{N+2} u_{k}(t) \mathrm{F}_{k}^{\beta}(0)\right)=\sum_{k=0}^{N+1}(-1)^{k} \mathfrak{u}_{k}(t) \quad \text { and } \quad \mathrm{u}_{\mathrm{N}}(1, t)=\sum_{k=0}^{N+2} u_{k}(t) F_{k}^{\beta}(1)\right)=\sum_{k=0}^{N+1} u_{k}(t),
$$

which implies that

$$
A_{4} U(t)=\left[\begin{array}{l}
h_{1}(t) \\
h_{2}(t)
\end{array}\right]
$$

where $A_{4}=\left[\begin{array}{cccccc}-1 & 1 & -1 & 1 & \cdots & (-1)^{N+1} \\ 1 & 1 & 1 & 1 & \cdots & 1\end{array}\right]_{2 \times(N+2)}$. From systems (3.5) and (3.6), we obtain the following fractional system

$$
B_{1} D_{t}^{\alpha} U(t)-B_{2}(t) U(t)=R(t),
$$

where

$$
B_{1}=\left[\begin{array}{c}
A_{1} \\
0_{2 \times(N+2)}
\end{array}\right], \quad B_{2}(t)=\left[\begin{array}{c}
A(t) A_{2} A_{3} \\
A_{4}
\end{array}\right], R(t)=\left[\begin{array}{c}
F(t) \\
h_{1}(t) \\
h_{2}(t)
\end{array}\right] .
$$

From equations (1.2) and (3.1), we see that

$$
g(x)=u_{N}(x, 0)=\sum_{k=0}^{N+2} u_{k}(0) F_{k}^{\beta}(x) .
$$

Using the orthogonality property of the fractional-order Legendre function, we get

$$
u_{k}(0)=\frac{\int_{0}^{1} g(x) F_{k}^{\beta}(x) d x}{\int_{0}^{1}\left(F_{k}^{\beta}(x)\right)^{2} d x}=(2 k+1) \beta \int_{0}^{1} g(x) \mathcal{L}_{k}(x) d x, \quad \text { for } k=0: N+2 .
$$


Therefore, our initial fractional value problem becomes

$$
\begin{aligned}
\mathrm{B}_{1} \mathrm{D}_{\mathrm{t}}^{\alpha} \mathrm{U}(\mathrm{t})-\mathrm{B}_{2}(\mathrm{t}) \mathrm{U}(\mathrm{t}) & =\mathrm{R}(\mathrm{t}), \\
\mathrm{u}(0) & =\mathrm{B},
\end{aligned}
$$

where

$$
B=\left[\begin{array}{c}
\beta \int_{0}^{1} g(x) \mathcal{L}_{0}(x) d x \\
3 \beta \int_{0}^{1} g(x) \mathcal{L}_{1}(x) d x \\
\vdots \\
(2 N+5) \int_{0}^{1} g(x) \mathcal{L}_{N+2}(x)(x) d x
\end{array}\right] .
$$

To solve the system (3.8)-(3.9), we use the fractional-order Legendre collocation method. Approximate the solution $\mathrm{U}(\mathrm{t})$ in terms of the fractional-order Legendre function as follows:

$$
v_{M}(t)=\sum_{k=0}^{M+1} u_{k} F_{k}^{\alpha}(t)
$$

Thus,

$$
D_{t}^{\alpha} V_{M}(x, t)=\sum_{k=0}^{M} u_{k}^{(\alpha)}(t) F_{k}^{\alpha}(t)
$$

where $u_{k}^{(\alpha)}=\sum_{j=k+1}^{M+1} a_{j k} u_{j}$, and $a_{j k}=(2 k+1) \alpha \int_{0}^{1} D^{\alpha} F_{j}^{\alpha}(t) F_{k}^{\alpha}(t) w(t) d t$, for $k=0,1,2, \ldots, M$ and $j=$ $k+1, k+2, \ldots, M+1$. Therefore, for $V_{M}(t)$, the residual is given by

$$
R\left(V_{M}(t)\right) \approx B_{1} D_{t}^{\alpha} V_{M}(t)-B_{2} V_{M}(t)-R(t) .
$$

Orthogonalize the residual with respect to the Dirac delta function as follows:

$$
<R\left(V_{M}(t)\right), \delta\left(t-t_{j}\right)>=\int_{0}^{1} R\left(V_{M}(t)\right) \delta\left(t-t_{j}\right) d t=0, \quad \text { for } j=0: M,
$$

where $t_{j}$ are the collocation points. We choose the collocation points to be

$$
\left\{\left(\frac{t_{0}+1}{2}\right)^{-\alpha},\left(\frac{t_{1}+1}{2}\right)^{-\alpha}, \ldots,\left(\frac{t_{M}+1}{2}\right)^{-\alpha}\right\}
$$

where $t_{0}, t_{1}, \ldots, t_{M}$ are the roots of $\mathcal{L}_{M+1}^{\prime}(t)$. Therefore, equation (3.10) leads to the elementwise equation

$$
B_{1} D_{t}^{\alpha} V_{M}\left(t_{j}\right)-B_{2}\left(t_{j}\right) V_{M}\left(t_{j}\right)-R\left(t_{j}\right)=0, \quad \text { for } j=0: M
$$

or

$$
B_{1} \sum_{k=0}^{M} u_{k}^{(\alpha)}(t) F_{k}^{\alpha}(t)-B_{2}\left(t_{j}\right) \sum_{k=0}^{M+1} u_{k} F_{k}^{\alpha}(t)-R\left(t_{j}\right)=0, \quad \text { for } j=0: M
$$


Let

$$
\mathrm{V}=\left[\begin{array}{c}
\mathrm{u}_{0} \\
\mathrm{u}_{1} \\
\vdots \\
\mathrm{U}_{\mathrm{M}^{\sim}+1}
\end{array}\right], \quad \mathrm{V}^{(\alpha)}=\left[\begin{array}{c}
\mathrm{u}_{0}^{(\alpha)} \\
\mathrm{u}_{1}^{(\alpha)} \\
\vdots \\
\mathrm{u}_{M}^{(\alpha)}
\end{array}\right], \quad \Lambda=\left[\begin{array}{c}
\mathrm{R}\left(\mathrm{t}_{0}\right) \\
\mathrm{R}\left(\mathrm{t}_{1}\right) \\
\vdots \\
\mathrm{R}\left(\mathrm{t}_{\mathrm{M}}\right)
\end{array}\right]
$$

Thus, we can rewrite Equation (3.3) in the matrix form as

$$
\mathrm{C}_{3} \mathrm{C}_{1} \mathrm{~V}^{(\alpha)}-\mathrm{C}_{4} \mathrm{C}_{2} \mathrm{~V}-\Lambda=0,
$$

where

$$
\begin{aligned}
C_{1} & =\left[\begin{array}{cccc}
B_{1} & 0 & \ldots & 0 \\
0 & B_{1} & \ldots & 0 \\
\vdots & \vdots & \ddots & \vdots \\
0 & 0 & \ldots & B_{1}
\end{array}\right]_{(N+3)(M+1) \times(N+3)(M+1)} \\
C_{2} & =\left[\begin{array}{cccc}
B_{2}\left(t_{0}\right) & 0 & \ldots & 0 \\
0 & B_{2}\left(t_{1}\right) & \cdots & 0 \\
\vdots & \vdots & \ddots & \vdots \\
0 & 0 & \ldots & B_{2}\left(t_{M+1}\right)
\end{array}\right]_{(N+3)(M+2) \times(N+3)(M+2)} \\
C_{3} & =\left[\begin{array}{cccc}
F_{0}^{\alpha}\left(t_{0}\right) I_{N+3} & F_{1}^{\alpha}\left(t_{0}\right) I_{N+3} & \ldots & F_{M}^{\alpha}\left(t_{0}\right) I_{N+3} \\
F_{0}^{\alpha}\left(t_{1}\right) I_{N+3} & F_{1}^{\alpha}\left(t_{1}\right) I_{N+3} & \ldots & F_{M}^{\alpha}\left(t_{1}\right) I_{N+3} \\
\vdots & \vdots & \ddots & \vdots \\
F_{0}^{\alpha}\left(t_{M}\right) I_{N+3} & F_{1}^{\alpha}\left(t_{M}\right) I_{N+3} & \cdots & F_{M}^{\alpha}\left(t_{M}\right) I_{N+3}
\end{array}\right]_{(N+3)(M+1) \times(N+3)(M+1)}
\end{aligned}
$$

and

$$
\mathrm{C}_{4}=\left[\begin{array}{cccc}
\mathrm{F}_{0}^{\alpha}\left(\mathrm{t}_{0}\right) \mathrm{I}_{\mathrm{N}+3} & \mathrm{~F}_{1}^{\alpha}\left(\mathrm{t}_{0}\right) \mathrm{I}_{\mathrm{N}+3} & \ldots & \mathrm{F}_{M+1}^{\alpha}\left(\mathrm{t}_{0}\right) \mathrm{I}_{\mathrm{N}+3} \\
\mathrm{~F}_{0}^{\alpha}\left(\mathrm{t}_{1}\right) \mathrm{I}_{\mathrm{N}+3} & \mathrm{~F}_{1}^{\alpha}\left(\mathrm{t}_{1}\right) \mathrm{I}_{\mathrm{N}+3} & \ldots & \mathrm{F}_{M+1}^{\alpha}\left(\mathrm{t}_{1}\right) \mathrm{I}_{\mathrm{N}+3} \\
\vdots & \vdots & \ddots & \vdots \\
\mathrm{F}_{0}^{\alpha}\left(\mathrm{t}_{M}\right) \mathrm{I}_{\mathrm{N}+3} & \mathrm{~F}_{1}^{\alpha}\left(\mathrm{t}_{M}\right) \mathrm{I}_{\mathrm{N}+3} & \ldots & \mathrm{F}_{M+1}^{\alpha}\left(\mathrm{t}_{M}\right) \mathrm{I}_{\mathrm{N}+3}
\end{array}\right]_{(\mathrm{N}+3)(\mathrm{M}+1) \times(\mathrm{N}+3)(M+2)}
$$

Since $u_{k}^{(\alpha)}=\sum_{j=k+1}^{M+1} a_{j k} u_{j}$, it is easy to see that $V^{(\alpha)}=C_{5} V$, where

$$
A_{3}=\left[\begin{array}{ccccccc}
0 \mathrm{I}_{\mathrm{N}+3} & \mathrm{a}_{10} \mathrm{I}_{\mathrm{N}+3} & \mathrm{a}_{20} \mathrm{I}_{\mathrm{N}+3} & \mathrm{a}_{30} \mathrm{I}_{\mathrm{N}+3} & \ldots & \mathrm{a}_{M 0} \mathrm{I}_{\mathrm{N}+3} & \mathrm{a}_{(M+1) 0} \mathrm{I}_{\mathrm{N}+3} \\
0 \mathrm{I}_{\mathrm{N}+3} & 0 \mathrm{I}_{\mathrm{N}+3} & \mathrm{a}_{21} \mathrm{I}_{\mathrm{N}+3} & \mathrm{a}_{31} \mathrm{I}_{\mathrm{N}+3} & \ldots & \mathrm{a}_{M 1} \mathrm{I}_{\mathrm{N}+3} & \mathrm{a}_{(M+1) 1} \mathrm{I}_{\mathrm{N}+3} \\
0 \mathrm{I}_{\mathrm{N}+3} & 0 \mathrm{I}_{\mathrm{N}+3} & 0 \mathrm{I}_{\mathrm{N}+3} & \mathrm{a}_{32} \mathrm{I}_{\mathrm{N}+3} & \ldots & \mathrm{a}_{M 2} \mathrm{I}_{\mathrm{N}+3} & \mathrm{a}_{(M+1) 2} \mathrm{I}_{\mathrm{N}+3} \\
\vdots & \vdots & \vdots & \vdots & \ddots & \vdots & \vdots \\
0 \mathrm{I}_{\mathrm{N}+3} & 0 \mathrm{I}_{\mathrm{N}+3} & 0 \mathrm{I}_{\mathrm{N}+3} & 0 \mathrm{I}_{\mathrm{N}+3} & \ldots & \mathrm{a}_{M M} \mathrm{I}_{\mathrm{N}+3} & \mathrm{a}_{(M+1) M} \mathrm{I}_{\mathrm{N}+3}
\end{array}\right]
$$

and $a_{j k}=(2 k+1) \alpha \int_{0}^{1} D^{\alpha} F_{j}^{\alpha}(t) F_{k}^{\alpha}(t) w(t) d t$ for $k=0,1,2, \ldots, M$ and $j=k+1: M+1$. Therefore, system (3.4) becomes

$$
\mathrm{C}_{3} \mathrm{C}_{1} \mathrm{C}_{5} \mathrm{~V}-\mathrm{C}_{4} \mathrm{C}_{2} \mathrm{~V}-\Lambda=0
$$

Now, we study the initial condition on the variable $x$. From Equation (3.9), one can see that

$$
\mathrm{B}=\mathrm{U}(0)=\sum_{\mathrm{k}=0}^{\mathrm{M}+1} \mathrm{U}_{\mathrm{k}} \mathrm{F}_{\mathrm{k}}^{\beta}(0)=\sum_{\mathrm{k}=0}^{\mathrm{M}+1}(-1)^{\mathrm{k}} \mathrm{u}_{\mathrm{k}}
$$

which implies that 


$$
\mathrm{C}_{6} \mathrm{~V}=\mathrm{B},
$$

where $\mathrm{C}_{6}=\left[\begin{array}{llllll}-\mathrm{I}_{\mathrm{N}+3} & \mathrm{I}_{\mathrm{N}+3} & -\mathrm{I}_{\mathrm{N}+3} & \mathrm{I}_{\mathrm{N}+3} & \cdots & (-1)^{\mathrm{N}+1} \mathrm{I}_{\mathrm{N}+3}\end{array}\right]_{(\mathrm{N}+3) \times(\mathrm{N}+3)(\mathrm{M}+2)}$. From systems (3.5) and (3.6), we obtain the following linear system

$$
\Omega V=\Psi,
$$

where

$$
\Omega=\left[\begin{array}{c}
\mathrm{C}_{3} \mathrm{C}_{1} \mathrm{C}_{5}-\mathrm{C}_{4} \mathrm{C}_{2} \\
\mathrm{C}_{6}
\end{array}\right], \quad \Psi=\left[\begin{array}{c}
\Lambda \\
\mathrm{B}
\end{array}\right] .
$$

Finally, we use Mathematica to solve the above linear system.

\section{Numerical results}

In this section, we implement the proposed numerical technique for several examples which are presented below.

Example 4.1. Consider the fractional diffusion equation

$$
\begin{aligned}
D_{t}^{\alpha} u(x, t) & =D_{x}^{\beta} u(x, t), \quad x \in(0,1), t \in(0,1), 0<\alpha \leqslant 1,1<\beta \leqslant 2, \\
u(0, t) & =E_{\alpha}\left(-t^{\alpha}\right), u(1, t)=E_{\alpha}\left(-t^{\alpha}\right) E_{\beta}(-1), 0<t<1, \\
u(x, 0) & =E_{\beta}\left(-x^{\beta}\right), 0<x<1,
\end{aligned}
$$

where $E_{\gamma}(z)$ is the Mittag-Leffler function. It is easy to see that the exact solution is

$$
u(x, t)=E_{\alpha}\left(-t^{\alpha}\right) E_{\beta}\left(-x^{\beta}\right)
$$

since $D_{t}^{\alpha} E_{\alpha}\left(-t^{\alpha}\right)=-E_{\alpha}\left(-t^{\alpha}\right)$ and $D_{x}^{\beta} E_{\beta}\left(-x^{\beta}\right)=-E_{\beta}\left(-x^{\beta}\right)$ for $0<\alpha<1$ and $1<\beta \leqslant 2$. The approximate solutions generated by the proposed method and the exact solution are presented in Figure 1 , for different values of $\alpha, \beta$, and $N=M=12$.
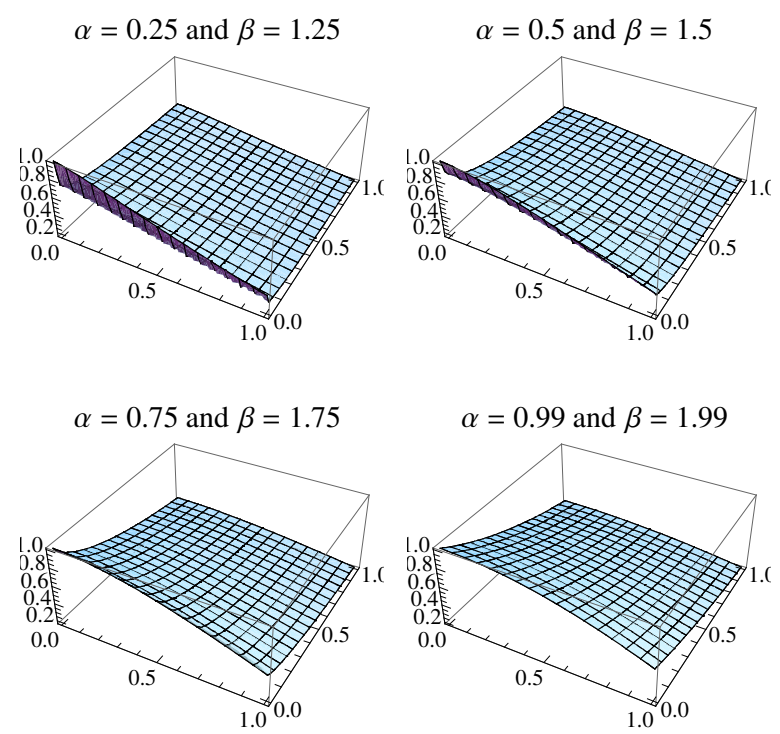

Figure 1: The exact and approximate solutions of Example 4.1.

Define the error

$$
\text { error }=\max \left\{\left|u\left(x_{i}, t_{j}\right)-u_{a p p}\left(x_{i}, t_{j}\right)\right|: x_{i}=0.01(i-1), t_{j}=0.01(j-1), i: 1: 101, j=1: 101\right\},
$$

where $u_{a p p}(x, t)$ is the approximate solution generated by the proposed method for $N=M=12$. Table 1 presents the error for different values of $\alpha$. 
Table 1: Error for Example 4.1.

\begin{tabular}{|l|l|l|}
\hline$\alpha$ & $\beta$ & error \\
\hline 0.5 & 1.5 & $5.7 * 10^{-10}$ \\
\hline 0.9 & 1.9 & $4.2 * 10^{-11}$ \\
\hline 0.99 & 1.99 & $2.1 * 10^{-11}$ \\
\hline 0.9999 & 1.9999 & $7.9 * 10^{-13}$ \\
\hline
\end{tabular}

Example 4.2. Consider the fractional diffusion equation

$$
\begin{aligned}
D_{t}^{\alpha} u(x, t) & =D_{x}^{\beta} u(x, t)-2 t^{3}+\frac{6 x^{3} t^{3-\alpha}}{\Gamma(4-\alpha)}-\frac{6 x^{3-\beta} t^{3}}{\Gamma(4-\beta)}, x \in(0,1), t \in(0,1), 0<\alpha \leqslant 1,1<\beta \leqslant 2, \\
u(0, t) & =0, u(1, t)=t^{3}, 0<t<1, \\
u(x, 0) & =0,0<x<1,
\end{aligned}
$$

with $u(x, t)=t^{3} x^{3}$ being the exact solution. The exact and approximate solutions generated by the proposed method are presented in Figure 2 for different values of $\alpha$ and $\beta$, and $N=M=8$. Figure 3 presents the exact solution $u(x, t)=t^{3} x^{3}$ and the approximate solution for $\alpha=1$ and $\beta=2$, and $\mathrm{N}=\mathrm{M}=8$.
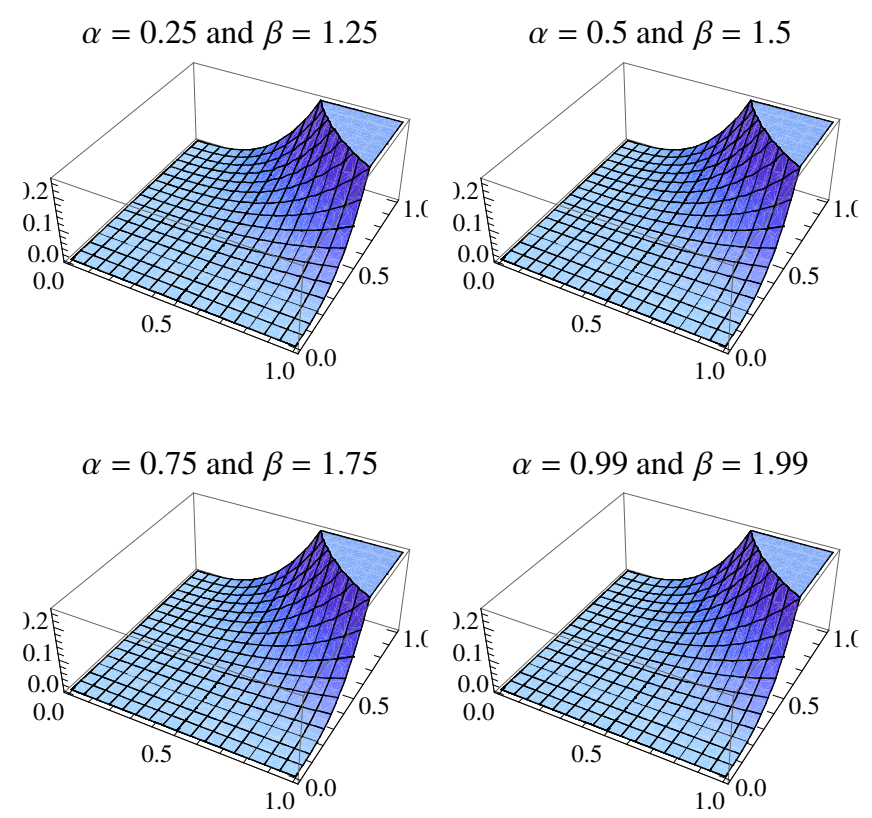

Figure 2: The exact and approximate solutions of Example 4.2.

Table 2: Error for Example 4.2.

\begin{tabular}{|l|l|l|}
\hline$\alpha$ & $\beta$ & error \\
\hline 0.25 & 1.25 & $9.1 * 10^{-13}$ \\
\hline 0.5 & 1.5 & $4.2 * 10^{-13}$ \\
\hline 0.75 & 1.75 & $8.6 * 10^{-14}$ \\
\hline 0.99 & 1.99 & $5.3 * 10^{-14}$ \\
\hline
\end{tabular}




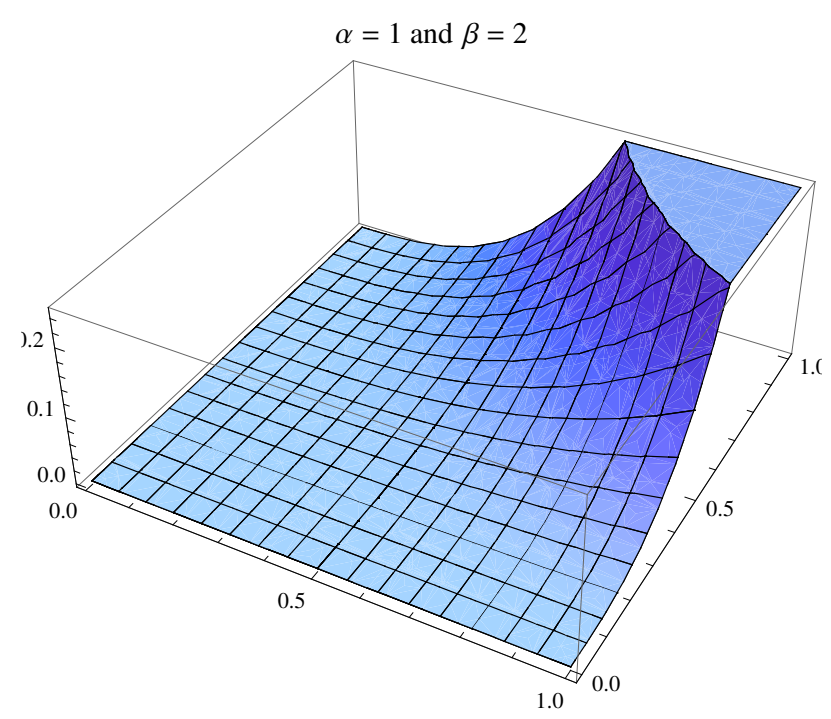

Figure 3: Exact and approximate solution for Example 4.2.

Example 4.3. Consider the fractional diffusion equation presented in [4],

$$
\begin{aligned}
D_{t}^{\alpha} u(x, t) & =k D_{x}^{\beta} u(x, t), x \in(0,1), t \in(0,1), 0<\alpha \leqslant 1,1<\beta \leqslant 2, \\
u(0, t) & =\frac{3 k t^{\alpha}}{\Gamma(1+\alpha)}, u(1, t)=\frac{3}{\Gamma(1+\alpha \beta)}+\frac{3 k t^{\alpha}}{\Gamma(1+\alpha)}, 0<t<1, \\
u(x, 0) & =\frac{3 x^{\beta}}{\Gamma(1+\beta)}, 0<x<1 .
\end{aligned}
$$

The solution of [4] and approximate solutions generated by the proposed method are presented in Figure 4 for $k=1, \alpha=0.8$ and $\beta=1.2$, and $N=M=6$. The approximate solutions generated by the proposed method are presented in Figure 5 for $k=1, \alpha=1$ and $\beta=2$, and $N=M=6$. Table 3 contains the absolute error between Gejji [4] and the proposed method for $k=1, \alpha=0.8$.

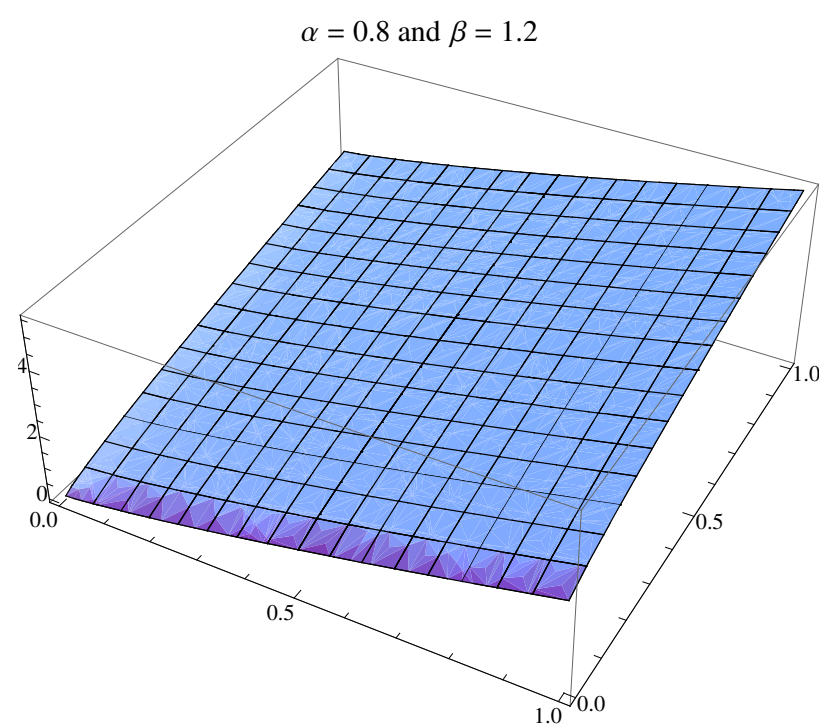

Figure 4: The approximate and [4] solutions for Example 4.3. 


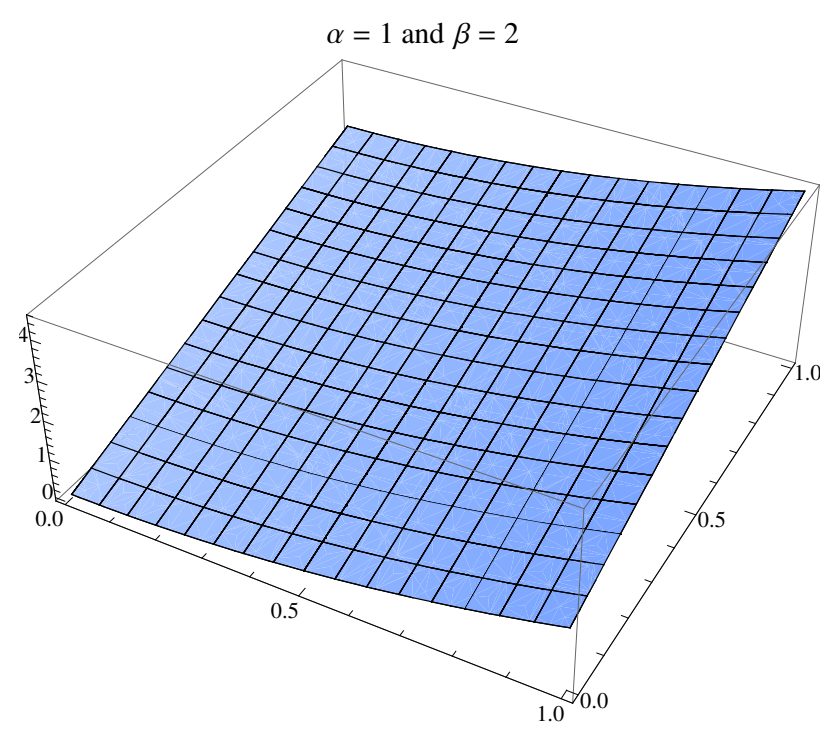

Figure 5: The approximate solution for Example 4.3.

Table 3: Absolute error of Gejji's method [4] and the proposed method.

\begin{tabular}{|l|l|l|l|}
\hline & \multicolumn{3}{|c|}{$\alpha=0.8$ and $\beta=1.2$} \\
\hline$x$ & Abs. Error $\mathrm{t}=0.4$ & Abs. Error $\mathrm{t}=0.8$ & Abs. Error $\mathrm{t}=0.9$ \\
\hline 0.1 & $4.6 * 10^{-16}$ & $1.7 * 10^{-16}$ & $3.1 * 10^{-16}$ \\
\hline 0.2 & $7.9 * 10^{-16}$ & $4.8 * 10^{-16}$ & $7.7 * 10^{-16}$ \\
\hline 0.3 & $1.3 * 10^{-15}$ & $1.1 * 10^{-15}$ & $1.5 * 10^{-15}$ \\
\hline 0.4 & $3.4 * 10^{-15}$ & $2.5 * 10^{-15}$ & $3.6 * 10^{-15}$ \\
\hline 0.5 & $6.1 * 10^{-15}$ & $4.9 * 10^{-15}$ & $5.7 * 10^{-15}$ \\
\hline 0.6 & $6.9 * 10^{-15}$ & $7.8 * 10^{-15}$ & $7.9 * 10^{-15}$ \\
\hline 0.7 & $8.9 * 10^{-15}$ & $9.2 * 10^{-15}$ & $9.4 * 10^{-15}$ \\
\hline 0.8 & $5.5 * 10^{-16}$ & $2.5 * 10^{-16}$ & $2.7 . * 10^{-16}$ \\
\hline 0.9 & $2.4 * 10^{-16}$ & $1.7 * 10^{-16}$ & $1.4 * 10^{-16}$ \\
\hline
\end{tabular}

\section{Conclusion}

In this paper, we use series expansion based on the fractional-order Legendre function to solve the generalized fractional diffusions equations of Caputo's type. We write the coefficients of the fractional derivative in terms of the shifted fractional Legendre functions as indicated in Theorem 2.5, and give explicit relationship between them. Then, we use the collocation method to compute these coefficients. To the best of our knowledge, the method has not been developed to integrate the generalize fractional diffusion equations of the form (1.1)-(1.2). We test the proposed technique for several examples, and present three of them in this paper. These examples show the efficiency and the accuracy of the proposed method, where in few terms we achieved high accuracy.

\section{Acknowledgment}

The authors would like to express their sincere appreciation to Taibah University for the financial support of grant no. 2543. 


\section{References}

[1] K. Al-Khaled, S. Momani, An approximate solution for a fractional diffusion-equation using the decomposition method, Appl. Math. Compu., 165 (2005), 473-483. 1

[2] Q. Al-Mdallal, M. Syam, M. N. Anwar, A collocation-shooting method for solving fractional boundary value problems, Commun. Nonlinear Sci. Numer. Simul., 15 (2010), 3814-3822. 1

[3] H. Beyer S. Kempfle, Definition of physically consistent damping laws with fractional derivatives, Z. Angew. Math. Mech., 75 (1995), 623-635. 1

[4] V. Daftardar-Gejji, S. Bhalekar, Solving fractional diffusion-wave equations using a new iterative method, Fract. Calc. Appl. Anal., 11 (2008), 193-202. 1, 4.3, 4, 3

[5] V. Daftardar-Gejji, S. Bhalekar, Solving multi-term linear and non-linear diffusion-wave equation of fractional order by Adomian decomposition method, Appl. Math. Comput., 202 (2008), 113-120. 1

[6] J.-H. He, Approximate analytical solution for seepage flow with fractional derivatives in porous media, Comput. Methods Appl. Mech. Engrg., 167 (1998), 57-68. 1

[7] J.-H. He, Some applications of nonlinear fractional differential equations and their approximations, Bull. Sci. Technol., 15 (1999), 86-90. 1

[8] M. Inc, The approximate and exact solutions of the space-and time-fractional Burger's equations with initial conditions by the variational iteration method, J. Math. Anal. Appl., 345 (2008), 476-484. 1

[9] H. Jafari, V. Daftardar-Gejji, Solving linear and nonlinear fractional diffusion and wave equations by Adomian decomposition, Appl. Math. Comput., 180 (2006), 488-497. 1

[10] S. Kazem, S. Abbasbandy, Sunil Kumar, Fractional-order Legendre functions for solving fractional-order differential equations, Appl. Math. Model., 37 (2013), 5498-5510. 1

[11] M. M. Khader, N. H. Sweilam, A. M. S. Mahdy, An efficient numerical method for solving the fractional diffusion equation, J. Appl. Math. Bioinformatics, 1 (2011), 1-12. 1

[12] Y. Luchko, Some uniqueness and existence results for the initial-boundary-value problems for the generalized time-fractional diffusion equation, Comput. Math. Appl., 59 (2010), 1766-1772. 1

[13] F. Mainardi, Fractals and Fractional Calculus in continuum mechanics, Springer-Verlag, 1997 (1997), 291-348. 1

[14] K. Moaddy, S. Momani, I. Hashim, The non-standard finite difference scheme for linear fractional PDEs in fluid mechanics, Comput. Math. Appl., 61 (2011), 1209-1216. 1

[15] Z. Obidat, S. Momani, The variational iteration method: and efficient scheme for handling fractional partial differential equations in fluid mechanics, Comput. Math. Appli., 58 (2009), 2199-2208. 1

[16] I. Podlubny, Fractional Differential Equations, Academic Press, San Diego, (1999). 2

[17] E. A. Rawashdeh, Legendre wavelets method for fractional integro-differential equations, Appl. Math. Sci. (Ruse), 5 (2011), 2467-2474. 1

[18] L. Song, H. Zhang, Application of homotopy analysis method to fractional KdV-Burgers-Kuramoto equation, Phys. Lett. A, 367 (2007), 88-94. 1

[19] N. H. Sweilam, M. M. Khader, R. F. Al-Bar, Numerical studies for a multi-order fractional differential equation, Phys. Lett. A, 371 (2007), 26-33. 1

[20] M. Syam, M. Al-Refai, Positive solutions and monotone iterative sequences for a class of higher order boundary value problems, J. Fract. Calculus Appl., 4 (2013), 1-13. 1, 2

[21] M. I. Syam, S. M. Al-Sharo', Collocation-continuation technique for solving nonlinear ordinary boundary value problems, Comput. Math. Appl., 37 (1999), 11-17. 2

[22] M. I. Syam, H. Siyyam, I. Al-Subaihi, Tau-Path following method for solving the Riccati equation with fractional order, J. Comput. Methods Phys., 2014 (2014). 7 pages. 2

[23] H. Xu, Analytical approximations for a population growth model with fractional order, Commun. Nonlinear Sci. Numer. Sumultat., 14 (2009), 1978-1983. 1

[24] C. Yang, J. Hou, An approximate solution of nonlinear fractional differential equation by Laplace transform and Adomian polynomials, J. Info. Comput. Sci., 10 (2013), 213-222.

[25] Y. Zhang, H. Ding, Finite differnece method for solving the time fractional diffusion equation, Communications in Computer and Information Science (AsiaSim 2012), 325 (2012), 115-123. 1 